\title{
Comparison of the effect of oxitropium bromide and of slow-release theophylline on nocturnal asthma
}

\author{
V. Bellia, G. Ferrara, F. Cibella, G. Cuttitta, A. Visconti, G. Insalaco, \\ M. Mirto and G. Peralta
}

Istituto di Pneumologia dell'Università, Istituto di Fisiopatologia Respiratoria del C.N.R., Palermo, Italy.

\begin{abstract}
Summary: The effects of a new inhaled antimuscarinic drug, oxitropium bromide, and of a slowrelease theophylline preparation upon nocturnal asthma were compared in a placebo-controlled double-blind study. Two samples were studied: 12 patients received oxitropium at $600 \mu \mathrm{g}$ (6 subjects) or at $400 \mu \mathrm{g}$ t.i.d. (6 subjects) whereas 11 received theophylline at $300 \mathrm{mg}$ b.i.d. Morning dipping, assessed by the fall in peak flow overnight, was significantly reduced in the periods when either active drug was taken, whereas no difference was noticed during the placebo administration. No significant difference was noticed between results obtained with either active drug, as well as with either dosage of oxitropium. No subject reported side effects of oxitropium, as compared to three subjects reporting nausea, vomiting and tremors after theophylline.

Oxitropium proves to be a valuable alternative to theophylline in nocturnal asthma, since it is equally potent, safer and does not require the titration of dosage.
\end{abstract}

\section{Introduction}

Nocturnal recurrence of attacks is a very common phenomenon among asthmatic subjects: ${ }^{1}$ its importance is related both to the resulting respiratory distress and to the possible unfavourable prognostic implications. ${ }^{2,3}$ The treatment of nocturnal asthma is mainly based upon slow-release theophylline preparations; ${ }^{4}$ however, there is some concern about the narrow therapeutic index of these drugs and the effects of physiological variables upon their kinetics: therefore a careful titration of the dosage is highly advisable in each patient.

Based upon some evidence of the role of vagal activity in producing nocturnal bronchoconstriction, ${ }^{5,6}$ some investigations have been recently carried out in order to evaluate the protective effect of antimuscarinic inhaled agents: both ipratropium bromide $^{7}$ and a longer acting derivative, oxitropium bromide, ${ }^{8}$ proved effective in protecting at least some 'responsive' patients.

The present investigation was aimed at comparing oxitropium bromide with the more conventional slow-release theophylline. In addition, since the effect of the antimuscarinic agent has been found to be dose-related, ${ }^{8}$ we evaluated also whether definitely high doses might be more effective than those used in this investigation. ${ }^{8}$

Correspondence: Professor V. Bellia M.D., Istituto di Pneumologia, Via Trabucco 180, 90146 Palermo, Italy. Accepted: 13 January 1988.
Materials and methods

\section{Patients}

Twenty-four asthmatic out-patients (13 males, 11 females) aged 18-60 years were selected on the basis of the following criteria: history of bronchial asthma as defined by the ACCP-ATS Joint Committee on Pulmonary Nomenclature; ${ }^{9}$ recent history of nocturnal asthma, defined as recurrent awakening with wheezing and breathlessness at night; ${ }^{10}$ early morning falls (morning dips) of peak expiratory flow (PEF) greater than $20 \%$ of the highest maximum daily value for at least 7 consecutive days preceding the study. Exclusion criteria were cardiovascular, ocular, genitourinary disease and a history of intolerance to theophylline or antimuscarinic agents.

Beta-stimulant drugs and corticosteroids were not administered throughout the study, whereas sodium cromoglycate was allowed, provided that no change in the administration regimen was introduced from the cited 7-day screening period till the end of the study.

\section{Treatments}

The following pharmaceutical preparations were used: (1) oxitropium bromide by metered aerosol

(C) The Fellowship of Postgraduate Medicine, 1988 
delivering $100 \mu \mathrm{g}$ per puff; (2) placebo of oxitropium by metered aerosol; (3) slow-release anhydrous theophylline in $300 \mathrm{mg}$ tablets; (4) placebo of theophylline in tablets.

\section{Protocol}

After the early run-in period, given the informed consent, subjects were randomly allocated to one of four treatment groups (Figure 1) so that on a double-blind basis everyone was tested with a single drug: in fact each subject alternated one of the active drugs (oral or inhaled) for one week and the relevant placebo for the other week, whereas the placebo of the other type of drug was administered throughout the whole 2-week period. The standard regimen for all the series was one tablet b.i.d. for the oral preparations; for the inhaled preparations, in order to allow a comparison between a medium and a high dosage, 12 randomly selected patients were instructed to inhale 4 puffs t.i.d., whereas the remaining 12 inhaled 6 puffs t.i.d.; in all cases the last daily dose was taken at bedtime, after performing the evening measurement of PEF (see below).

All subjects were recommended to keep a regular daily schedule of diurnal activity and nocturnal rest. They were instructed to record PEF four times daily (on waking, at lunch time, in the afternoon and at bedtime): on each occasion they made the measurement in triplicate and recorded the highest value on a diary chart. On the same chart they reported respiratory symptoms occurring at night or in the early morning (chest tightness, cough and wheeze), as well as any untoward effect possibly related to treatments.

At the end of each week of treatment, patients were submitted to clinical and functional assessment (forced expiratory volume in one second, $\mathrm{FEV}_{1}$ ), as well as to the measurement of the trough

\begin{tabular}{|c|c|c|c|c|c|}
\hline & \multicolumn{2}{|c|}{ Inhaled } & \multicolumn{2}{|c|}{ Oral } \\
\hline & & OB & POB & $\mathrm{T}$ & PT \\
\hline \multirow{2}{*}{\multicolumn{2}{|c|}{$\begin{array}{cl}\text { Group a } & \begin{array}{l}\text { st week } \\
2 \text { nd week }\end{array}\end{array}$}} & & & $=1$ & \\
\hline & & & & & \\
\hline \multirow{2}{*}{ Group b } & 1st week & & & & \\
\hline & 2 nd week & & & & \\
\hline \multirow{2}{*}{ Group c } & 1st week & $=-3=$ & & & \\
\hline & 2nd week & & & & \\
\hline \multirow{2}{*}{ Groupd } & 1st week & & & & \\
\hline & 2 nd week & I: & & & \\
\hline
\end{tabular}

Figure 1 Schematic diagram of the protocol of administration of drugs. OB: oxitropium bromide; POB: placebo of oxitropium bromide; T: theophylline; PT: placebo of theophylline. theophylline concentration (nephelometry, ICS, Beckman).

\section{Evaluation of results}

The effects of treatments were evaluated by measuring the morning to maximum PEF ratio of the last 5 days of each week of treatment, since the early 2 days were assumed as accounting for the wash-in/ wash-out period for any treatment. Statistical analysis was performed by Student's ' $t$ ' test for paired or unpaired data as applicable to various circumstances.

\section{Results}

Since one patient of Group b withdrew because of nausea and vomiting, the results of 23 patients were examined: 12 had been submitted to administration of oxitropium (6 at $400 \mu \mathrm{g}$ t.i.d. and 6 at $600 \mu \mathrm{g}$ t.i.d.), whereas 11 had been on theophylline. In the latter patients, trough theophylline concentrations were within the therapeutic range $(9.9$ to $13.7 \mathrm{mg} / \mathrm{l})$. No significant difference was recorded for $\mathrm{FEV}_{1}$ between the two samples at the beginning of the study $(81.1 \%$ predicted \pm 18.7 for oxitropium and $81.7 \%$ predicted \pm 12.1 for theophylline) and the mean morning dip of PEF over the run-in period (respectively $35.7 \%$ predicted \pm 12.1 and $37.3 \%$ predicted \pm 16.4 ).

After both treatments (Figure 2) a significant decrease in morning dip was recorded in all patients; in fact in $9 / 12$ of the oxitropium group (4 in the $600 \mu \mathrm{g}$ subgroup and 5 in that submitted to the lower dose regimen) and 8/11 of the theophylline group the index fell below the $20 \%$ threshold. Similar results were obtained for $\mathrm{FEV}_{1}$ (Figure 3).

No significant difference between results obtained with either active drug, as well as with either

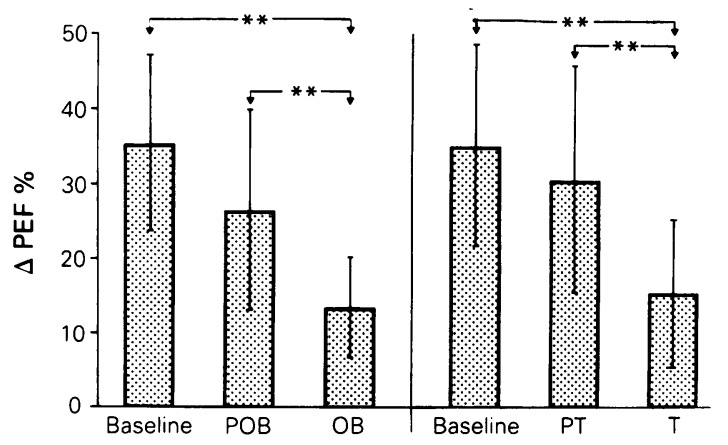

Figure 2 Effects of different treatments upon morning dip of PEF ( $\triangle \mathrm{PEF})$. For abbreviations see Figure 1. $* * P<0.01$. 


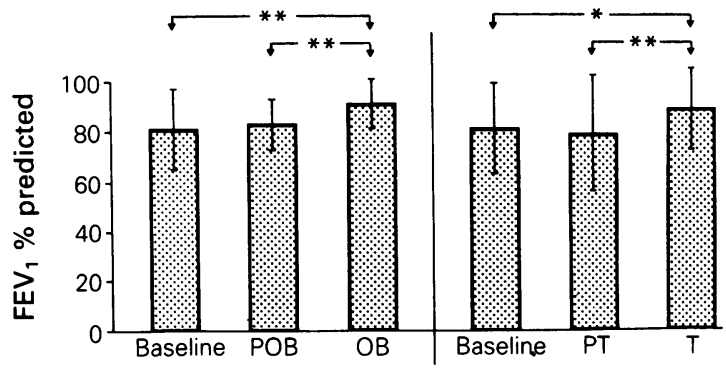

Figure 3 Effects of different treatments upon $\mathrm{FEV}_{1}$. For abbreviations see Figure 1. ${ }^{*} P<0.05 ;{ }^{* *} P<0.01$.

dosage of oxitropium was noticed. Finally, no significant difference between baseline values and the results of both placebos was found.

All subjects reported a subjective improvement when submitted to the active drug, as expressed by a reduction of events of nocturnal awakening with symptoms and by the disappearance of chest tightness in the early morning.

No significant side effects from oxitropium were recorded, whereas in three cases submitted to theophylline, nausea, vomiting and tremors were noticed.

\section{Discussion}

Oxitropium bromide has been demonstrated to be a long-acting antimuscarinic bronchodilator: in fact its effects have been reported as still significant 10 hours after inhalation. ${ }^{11,12}$ The results of the present investigation suggest that because of this characteristic it proves as effective as theophylline in preventing nocturnal asthma but with fewer side effects and without any need for titrating the dose in each patient.

The high therapeutic ratio of the antimuscarinic agent is further confirmed by the use of a definitely high dose (i.e., $600 \mu \mathrm{g}$ ), which proves to be as safe as the $400 \mu \mathrm{g}$ one: the comparable effects of the two dosages upon morning dip, if analysed along with the results of Coe and Barnes, ${ }^{8}$ concerning the use

\section{References}

1. Turner Warwick, M. The definition and recognition of nocturnal asthma. In: Barnes, P.J. \& Levy, J. (eds) Nocturnal Asthma. The Royal Society of Medicine, London, 1984, pp 3-5.

2. Hetzel, M.R., Clark, T.J.H. \& Branthwaithe, M.A. Analysis of sudden deaths and ventilatory arrests in hospital. Br Med J 1977, 1: 808-811.

3. Bateman, J.R.M. \& Clarke, S.W. Sudden death in asthma. Thorax 1979, 34: 40-44. of 200 and $400 \mu \mathrm{g}$, allow one to conclude that $400 \mu \mathrm{g}$ may be regarded as the optimal dose in the specific setting of nocturnal asthma and that further increases in dosage, though apparently safe, are not warranted by any further gain in therapeutic effect.

Unlike the previous investigation, ${ }^{8}$ no patient was found to be a non-responder to therapy, since the decrease in morning dip was significant in all patients, including those $3 / 12$ in whom the index did not fall below the $20 \%$ threshold, commonly considered as associated with nocturnal asthma. Anyway a comparable proportion of subjects on theophylline showed a similar behaviour, thus once more confirming the similar spectrum of the two drugs.

Since no apparent difference in clinical characteristics of samples may be pointed out, this difference with respect to the previous investigation ${ }^{8}$ may be interpreted as due to the fact that in the previous study patients were given a single dose at night, whereas in our investigation they were submitted to regular administration throughout the day: on this basis it is possible that a more effective control of vagal activity was obtained, decreasing the baseline vagal tone and thus further reducing the effects of variations in vagal drive or in vagal efferent activities occurring during the night.

Though grounded on physiological as well as once experimental evidence, the use of inhaled anti-? cholinergic drugs has not yet gained a wide acceptance, probably because of prejudice derived from the early experiences with atropine compounds. The results of the present investigation warrant a preliminary therapeutic challenge with these drugs in all patients affected by nocturnal asthma. With the exception of definite contraindications, the use of oxitropium bromide may prove safer than that of theophylline and at least equally effective.

\section{Acknowledgement}

Boehringer-Ingelheim - Istituto De Angeli kindly supplied the oxitropium bromide.

4. Isles, A.F. \& Von Wichert, P. (eds) In: Sustained Release Theophylline and Nocturnal Asthma. Excerpta Medica, Amsterdam, 1985.

5. Postma, D.S., Keyzer, J.J., Koeter, G.H., Sluiter, H.J. \& De Vries, $K$. Influence of the parasympathetic nervous system on nocturnal bronchial obstruction. Clin Sci 1985, 69: 251-258. 
6. Kallenbach, J.M., Webster, T., Dowdeswell, R., Reinach, S.G., Scott Millar, R.N. \& Zwi, S. Reflex heart rate control in asthma. Chest 1985, 87: 644-648.

7. Cox, I.D., Hughes, D.T.D. \& McDonnell, K.A. Ipratropium bromide in patients with nocturnal asthma. Postgrad Med J 1984, 60: 526-528.

8. Coe, C.I. \& Barnes, P.J. Reduction of nocturnal asthma by an inhaled anticholinergic drug. Chest 1986, 90: 485-488.

9. ACCP-ATS Joint Committee on Pulmonary Nomenclature. Pulmonary terms and symbols. Chest 1987, 67: 583-593.
10. Hughes, D.T.D. The use of anticholinergic drugs in nocturnal asthma. Postgrad Med J 1987, 63 (Suppl. 1): 47-51.

11. Peel, E.T., Anderson, G., Cheong, B. \& Broderick, N. A comparison of oxitropium bromide and ipratropium bromide in asthma. Eur J Respir Dis 1984, 65: 106-108.

12. Kneubühler, H.R., Kyd, K., Benoit, R.C. \& Scherrer, M. Das neue anticholinergische Bronchospasmolytikum Oxitropiumbromid. Sein lang anhaltender Schutz über die Nacht hinweg. Schweiz med Wschr 1980, 110: 812-816.

(0) 